\title{
Optical bistability in lasers induced by active molecules with a large permanent dipole moment
}

\author{
Oscar G. Calderón, ${ }^{1}$ Sonia Melle, ${ }^{2}$ and Isabel Gonzalo ${ }^{1}$ \\ ${ }^{1}$ Departamento Optica, Universidad Complutense, Ciudad Universitaria s/n, 28040 Madrid, Spain \\ ${ }^{2}$ Departamento Física Fundamental, UNED. Paseo Senda del Rey 9, 28040 Madrid, Spain
}

(Received 15 June 2001; published 15 January 2002)

\begin{abstract}
We study a single-mode laser system, whose active medium consists of molecules with a large difference between excited and ground electrical permanent-dipole moments. In this case, the Maxwell-Bloch equations are further coupled by nonlinear terms involving the ratio between this difference between the dipoles and the transition dipole moment. It is found that these new terms lead to multiple stationary solutions. From the linear stability analysis, we demonstrate the bistable (or multistable) character of the lasing solutions.
\end{abstract}

DOI: 10.1103/PhysRevA.65.023811 PACS number(s): 42.55.-f, 42.65.Pc, 42.70.Hj, 05.45.-a

\section{INTRODUCTION}

In the theoretical description of the interaction of an electromagnetic field with a multilevel molecular system, it is common practice to ignore the presence of electric permanent-dipole moments. However, there are many systems where the permanent dipoles must be considered. In a strongly excited two-level molecular system, the nonzero difference between the electric permanent dipoles leads to appealing phenomena: sharp features appearance in onresonant one-photon or two-photon nonlinear absorption and dispersion [1-4]; Raman scattering [5]; high-reflectivity two-photon phase conjugation [6,7]; emergence of additional resonances on nondegenerate four-wave mixing [8]; direct two-photon transitions in a two-level instead of a multilevel system [1,9]; microwave amplification through laser radiation [10]; high-molecular transparency to optical waves of certain frequencies [11]; and high-harmonic generation $[12,13]$.

Kothari and Kobayashi [1] and Kobayashi, Kothari, and Uchiki [14] found that two-photon optical bistability can result in small cavities if the nonlinear medium is composed of molecules with a large difference between the excited and ground electric permanent-dipole moments. Optical bistability has received considerable attention from both theoretical and experimental points of view because of its potential applications in signal processing. Many different optical systems, such as lasers without inversion [15] and Raman lasers [16], can exhibit bistable behavior under certain excitation conditions. In previous works $[17,18]$, it was shown that the electronic-vibrational coupling in molecules can produce optical bistability in lasers.

We study a single-mode laser system whose active molecules present a large difference between excited and ground electrical permanent-dipole moments. We follow a semiclassical treatment by means of the Maxwell-Bloch equations. The rotating wave and the slowly varying amplitude approximations are assumed. It is found that the new terms appearing in the Maxwell-Bloch equations can lead to multiple stationary solutions. The linear stability analysis is carried out, and shows that positive-slope regions of the transmission curves (in our case, pump versus laser intensity) are stable. Permanent-dipole moments can then induce multistability in lasers.
In Sec. II, we describe the procedure to obtain the evolution equations of the density matrix and the radiation field, i.e., the Maxwell-Bloch equations for our system. In Sec. III, we analyze the dynamics of our system and present a numerical example. Finally, we briefly conclude in Sec. IV.

\section{THE MODEL}

We consider the active medium of the laser composed of $N$ identical noncentrosymmetric molecules per unit volume in a cavity. The length of the cavity $L$ is tuned to a single longitudinal mode of frequency $\omega$. As usual in laser theory, the active medium is considered as a two-level system with population inversion. This population inversion is created by incoherent pumping (electrical or flashlamp, rf discharge, collisions, etc.) and is introduced phenomenologically in the matter laser equations. For each molecule, we consider the ground-state $|1\rangle$ with energy $E_{1}$ and the excited-state $|2\rangle$ with energy $E_{2}$. The levels have unequal electric-dipole moments and are connected by one-photon transitions, so that $\hbar \omega$ $\approx E_{2}-E_{1}$.

The Hamiltonian in the electric-dipole approximation is

$$
H=H^{0}+H^{e-r},
$$

where $H^{0}$ is the free electronic Hamiltonian, whose eigenstates are $|1\rangle$ and $|2\rangle$. The electron-radiation interaction Hamiltonian $H^{e-r}=-\vec{\mu} \cdot \vec{E}$, where $\vec{\mu}$ is the electric-dipole moment. We consider that the active molecules are oriented in the same direction. It occurs, for example, in some molecular crystals or when the molecules are inserted in a solid matrix that is transparent to the radiation. For the sake of simplicity, the transition dipole moment and the difference between the permanent-dipole moments are assumed to be parallel to the radiation field $\vec{E}$. As stated in Ref. [2], in the case of freely rotating molecules, averaged physical quantities should be taken into account. Even in that case, the effects of permanent dipoles do not vanish but they are reduced to some extent. The problem of the freely rotating molecules is very complicated and we limit the discussion in this paper to the case of rigid molecular system.

The electronic state of the molecular system can be expressed as 


$$
|\psi(t)\rangle=C_{1}(t) e^{-i E_{1} t / \hbar}|1\rangle+C_{2}(t) e^{-i E_{2} t / \hbar}|2\rangle .
$$

Let us write the total Hamiltonian in matrix form, with the choice $\left(\begin{array}{l}1 \\ 0\end{array}\right)$ for the excited-state $|2\rangle$, and $\left(\begin{array}{l}0 \\ 1\end{array}\right)$ for the groundstate $|1\rangle$,

$$
H=\left(\begin{array}{cc}
E_{2}-\mu_{22} E & -\mu_{21} E \\
-\mu_{12} E & E_{1}-\mu_{11} E
\end{array}\right),
$$

where $\mu_{i j} \equiv\langle i|\mu| j\rangle$. We can always choose $\mu_{12}$ real, such that $\mu_{21}=\mu_{12}$. Note that the permanent dipoles $\mu_{22}$ and $\mu_{11}$ are in the diagonal terms. The expression of $H$ written in terms of the Pauli matrices reads

$$
\begin{aligned}
H= & \frac{1}{2}\left[E_{2}+E_{1}-\left(\mu_{22}+\mu_{11}\right) E\right] \sigma_{0}+\frac{1}{2}\left(\hbar \omega_{21}-\mu_{p} E\right) \sigma_{3} \\
& -\mu_{12} E\left(\sigma_{+}+\sigma_{-}\right),
\end{aligned}
$$

where $\mu_{p} \equiv \mu_{22}-\mu_{11}, \omega_{21} \equiv\left(E_{2}-E_{1}\right) / \hbar, \sigma_{0}$ is the unit matrix, and the Pauli matrices verify the commutation relations $\left[\sigma_{ \pm}, \sigma_{3}\right]=\mp 2 \sigma_{ \pm}$and $\left[\sigma_{+}, \sigma_{-}\right]=\sigma_{3}$.

The aim of our development is to solve the evolution equation for the density matrix, $i \hbar \partial \rho / \partial t=[H, \rho]$ $+i \hbar(\partial \rho / \partial t)_{\text {rel }}$. The last term, introduced phenomenologically, takes into account the coherence and population relaxation rates. Now we follow the same procedure as in $[9,13]$, which consists of applying a series of unitary transformations in order to solve the evolution equation involving the permanent dipoles, in a simple way. If $U$ is a unitary transformation and $\rho^{(T)}=U \rho U^{-1}$, then

$$
i \hbar \frac{\partial \rho^{(T)}}{\partial t}=\left[H^{(T)}, \rho^{(T)}\right]+i \hbar\left(\frac{\partial \rho^{(T)}}{\partial t}\right)_{\text {rel }},
$$

where

$$
H^{(T)} \equiv U H U^{-1}+i \hbar \frac{\partial U}{\partial t} U^{-1}
$$

The term $i \hbar\left(\partial \rho^{(T)} / \partial t\right)_{\text {rel }}$ accounts for the transformed coherence and population relaxation rates and is added phenomenologically. Three of these unitary transformations are applied in the following succession. The first one, $U_{0}$, is used to eliminate the term with $\sigma_{0}$ in the Hamiltonian of Eq. (4). It reads

$$
U_{0}=\exp \left(\eta \sigma_{0}\right)
$$

with

$$
\eta \equiv \frac{i}{2 \hbar}\left[\left(E_{2}+E_{1}\right) t-\left(\mu_{22}+\mu_{11}\right) \int_{0}^{t} E\left(z, t^{\prime}\right) d t^{\prime}\right],
$$

where the integration time $t$ is assumed to be much smaller than the coherence and population decay times. Applying the transformation $U_{0}$ to the Hamiltonian of Eq. (4), we obtain from Eq. (6),

$$
H^{(0)}=\frac{1}{2}\left(\hbar \omega_{21}-\mu_{p} E\right) \sigma_{3}-\mu_{12} E\left(\sigma_{+}+\sigma_{-}\right) .
$$

With a second unitary transformation, the explicit temporal dependence introduced by the field $E$ in the diagonal elements can be eliminated. This transformation is defined as

$$
U_{1}=\exp \left(\xi \sigma_{3}\right)
$$

with

$$
\xi \equiv \frac{i}{2 \hbar}\left[\hbar \omega_{21} t-\mu_{p} \int_{0}^{t} E\left(z, t^{\prime}\right) d t^{\prime}\right] .
$$

In a similar way to the preceding case, we apply the transformation $U_{1}$ to the Hamiltonian of Eq. (9), and using $U_{1} \sigma_{ \pm} U_{1}^{-1}=e^{ \pm 2 \xi} \sigma_{ \pm}$, we obtain

$$
H^{(1)}=-\mu_{12} E\left(\sigma_{+} e^{2 \xi}+\sigma_{-} e^{-2 \xi}\right)=-\mu_{12} E\left(\begin{array}{cc}
0 & e^{2 \xi} \\
e^{-2 \xi} & 0
\end{array}\right) \text {. }
$$

Before applying the third transformation, let us first evaluate these off-diagonal elements. The radiation field inside the medium can be expressed as

$$
E(z, t)=E_{0}^{+}(z, t) e^{-i(\omega t-k z)}+\text { c.c., } E_{0}^{(+)}=\frac{1}{2} E_{0} e^{i \varphi},
$$

where c.c. denotes complex conjugation, $E_{0}$ is the field amplitude, and $\varphi$ its phase. Inserting the above expression of the field in Eq. (11) and carrying out the integral, we obtain from Eq. (12),

$$
\begin{aligned}
H_{12}^{(1)}= & -\mu_{12} E e^{-2 \xi}=-\mu_{12} E \exp \left(-i \omega_{21} t+i \frac{\mu_{p} E_{0}}{\hbar \omega}\right. \\
& \left.\times \sin (\omega t-k z-\varphi)+i \frac{\mu_{p} E_{0}}{\hbar \omega} \sin (k z+\varphi)\right) .
\end{aligned}
$$

Making use of $\exp (i q \sin \alpha)=\sum_{t=-\infty}^{\infty} J_{l}(q) \exp (i l \alpha)$, where $J_{l}(q)$ is the $l$-order Bessel function, Eq. (14) becomes

$$
\begin{aligned}
H_{12}^{(1)}= & -\frac{\mu_{12}}{2} E_{0} \exp (i q \sin (k z+\varphi))\left[\sum_{l=-\infty}^{\infty} J_{l}(q) \exp [i(l+1)\right. \\
& \left.\times(\omega t-k z-\varphi)-i \omega_{2} t\right]+\sum_{l=-\infty}^{\infty} J_{l}(q) \exp [i(l-1) \\
& \left.\left.\times(\omega t-k z-\varphi)-i \omega_{21} t\right]\right]
\end{aligned}
$$

where the argument $q \equiv \mu_{p} E_{0} /(\hbar \omega)$. At this point, we apply the rotating-wave approximation. Assuming the one-photon resonance condition $\omega \simeq \omega_{21}$, we only retain the terms with $l=0$ in the first series and the terms with $l=2$ in the second series. Equation (15) can then be written as

$$
H_{12}^{(1)}=-\mu_{\mathrm{eff}}\left(E_{0}^{(+)}\right) * e^{i \Phi},
$$

where

$$
\Phi \equiv q \sin (k z+\varphi)-k z-\left(\omega_{21}-\omega\right) t
$$


and

$$
\mu_{\mathrm{eff}} \equiv \mu_{12}\left[J_{0}(q)+J_{2}(q)\right]=\mu_{12} \frac{2 J_{1}(q)}{q}
$$

is the effective transition dipole moment for the two-level transformed system. It is field dependent and involves the difference between the permanent-dipoles $\mu_{p}$. Note that for values of $q$, making $J_{1}$ zero, the transition is inhibited while it is enhanced for values of $q$ making $J_{1}$ maximum. In the case of equal permanent-dipole moments, $\mu_{p}=0$, the effective dipole reduces to the usual transition dipole moment $\mu_{12}$, as expected.

In order to eliminate the explicit temporal dependence in the elements of $H^{(1)}$, we consider the third unitary transformation

$$
U_{2}=\exp \left(i \frac{\Phi}{2} \sigma_{3}\right)
$$

where $\Phi$ is given by Eq. (17). Following a procedure analogous to that followed for the other transformations, the Hamiltonian $H^{(1)}$ becomes

$$
H^{(2)}=\left(\begin{array}{cc}
\frac{\hbar}{2}\left(\omega_{21}-\omega\right) & -\omega_{\mathrm{eff}} E_{0}^{(+)} \\
-\mu_{\mathrm{eff}}\left(E_{0}^{(+)}\right)^{*} & -\frac{\hbar}{2}\left(\omega_{21}-\omega\right)
\end{array}\right) .
$$

The transformed density matrix then satisfies $i \hbar \partial \rho^{(2)} / \partial t$ $=\left[H^{(2)}, \rho^{(2)}\right]+i \hbar\left(\partial \rho^{(2)} / \partial t\right)_{\text {rel }}$, and the evolution equations of the molecular system read

$$
\begin{gathered}
\frac{\partial \rho_{21}^{(2)}}{\partial t}=-\gamma_{\perp} \rho_{21}^{(2)}-i\left(\omega_{21}-\omega\right) \rho_{21}^{(2)}-i \frac{\mu_{\mathrm{eff}}}{\hbar} E_{0}^{(+)} \rho_{D}^{(2)} \\
\frac{\partial \rho_{D}^{(2)}}{\partial t}=-\gamma_{\|}\left[\left(\rho_{D}^{(2)}-\rho_{D 0}\right)+i \frac{2 \mu_{\mathrm{eff}}}{\hbar}\left[E_{0}^{(+)} \rho_{12}^{(2)}\right.\right. \\
\left.\left.-\left(E_{0}^{(+)}\right)^{*} \rho_{21}^{(2)}\right]\right]
\end{gathered}
$$

where $\rho_{D}^{(2)} \equiv \rho_{22}^{(2)}-\rho_{11}^{(2)}$ is the population inversion. The stationary value of $\rho_{D}$ in absence of radiation is $\rho_{D}=\rho_{D 0}$, where $\rho_{D 0}$ is the population inversion induced by the pumping. Note that the diagonal terms of the density matrix remain invariant under the applied transformations $\left(\rho_{D}\right.$ $\left.=\rho_{D}^{(2)}\right)$. The quantities $\gamma_{\|}^{-1}$ and $\gamma_{\perp}^{-1}$ are the population (diagonal) and coherent (off-diagonal) relaxation times, respectively. They are introduced phenomenologically.

The response of the medium to an electromagnetic field is characterized by the induced polarization $P(z, t)$, which acts as a source term in the Maxwell wave equation. The polarization is given by

$$
P(z, t)=N \operatorname{Tr}(\mu \rho)=N\left[\mu_{22} \rho_{22}+\mu_{11} \rho_{11}+\mu_{12}\left(\rho_{21}+\rho_{12}\right)\right] \text {. }
$$

The matrix elements of $\rho$ can be expressed in terms of those of $\rho^{(2)}$ by applying the inverse transformations, $\rho$ $=U_{0}^{-1} U_{1}^{-1} U_{2}^{-1} \rho^{(2)} U_{2} U_{1} U_{0}$. Disregarding the static polarization, we obtain for the oscillating contribution,

$$
\begin{aligned}
P(z, t) \equiv & N\left[\mu_{12}\left(\rho_{21}+\rho_{12}\right)\right] \\
= & N \mu_{12} \rho_{21}^{(2)} \exp (-2 \xi-i \Phi)+\text { c.c. } \\
= & N \mu_{12} \rho_{21}^{(2)}\left\{\sum_{l=-\infty}^{\infty} J_{l}(q) \exp [i(l-1)(\omega t-k z)]\right. \\
& \times \exp (-i \varphi l)\}+ \text { c.c. }
\end{aligned}
$$

The polarization of a molecular system with permanentdipole moments can then oscillate at frequencies $\omega, 2 \omega, 3 \omega$, etc., provided that a radiation field of frequency $\omega$ interacts with it. Here, we are interested in the polarization oscillating at frequency $\omega$. This polarization can be written as $P^{(1)}(z, t)=P_{0}^{+}(z, t) \exp \{-i(\omega t-k z)\}+$ c.c., where $P_{0}^{+}(z, t)$ is the slowly varying amplitude of the polarization. This amplitude can be obtained from Eq. (24) by retaining the terms with $l=0$ and 2 , that is,

$$
P_{0}^{(+)}(z, t)=N \mu_{12}\left[\rho_{21}^{(2)} J_{0}(q)+\rho_{12}^{(2)} J_{2}(q) \exp (2 i \varphi)\right] \text {. }
$$

The behavior of the radiation inside the medium is described by the Maxwell wave equation which, under the slowly varying amplitude approximation and leaving out the transversal character of the radiation field, can be written as

$$
\frac{\partial E_{0}^{+}}{\partial t}=-\kappa E_{0}^{(+)}+\frac{i \omega}{2 \epsilon_{0}} P_{0}^{(+)}
$$

where $P_{0}^{+}(z, t)$ is given by Eq. (25) and $\kappa$ stands for the effective cavity losses. To obtain dimensionless and simplified equations, we define the dimensionless detuning $\Delta$ $\equiv\left(\omega_{21}-\omega\right) / \gamma_{\perp}$, the normalized pump $r \equiv N \omega \mu_{12}^{2} \rho_{D 0} /$ $\left(2 \hbar \epsilon_{0} \gamma_{\perp} \kappa\right)$, and the following variable changes:

$$
\begin{gathered}
E_{0}^{(+)}=\frac{\hbar \sqrt{\gamma_{\|} \gamma_{\perp}}}{2 \mu_{12}} E_{s}, \\
\rho_{21}^{(2)}=-\frac{i}{2} \sqrt{\frac{\gamma_{\|}}{\gamma_{\perp}}} \frac{\rho_{D 0}}{r} P_{s}, \\
\rho_{D}^{(2)}=\frac{\rho D_{0}}{r} D_{s},
\end{gathered}
$$

where $E_{s}, P_{s}$, and $D_{s}$ are the dimensionless magnitudes. In terms of these variables, Eqs. (21), (22), and (26) finally can be written as

$$
\frac{\partial E_{s}}{\partial t}=-\kappa\left[E_{s}-J_{0}(q) P_{s}+J_{2}(q) P_{s}^{*} e^{2 i \varphi}\right]
$$




$$
\begin{gathered}
\frac{\partial P_{s}}{\partial t}=-\gamma_{\perp}\left[(1+i \Delta) P_{s}-\left[J_{0}(q)+J_{2}(q)\right] D_{s} E_{s}\right], \\
\frac{\partial D_{s}}{\partial t}=-\gamma_{\|}\left[D_{s}-r+\frac{\left[J_{0}(q)+J_{2}(q)\right]}{2}\left(E_{s}^{*} P_{s}+E_{s} P_{s}^{*}\right)\right],
\end{gathered}
$$

where $\left.\quad q=\mu_{p} E_{0} /(\hbar \omega)=\beta \mid E_{s}\right), \quad$ with $\quad \beta \equiv\left(\mu_{p} /\right.$ $\left.\mu_{12}\right)\left(\sqrt{\gamma_{\perp} \gamma_{\|}} / \omega\right)$. These equations are similar to the complex Lorenz equations except for the nonlinear field dependence due to the Bessel functions.

\section{DYNAMICS OF THE SYSTEM}

In order to simplify the analysis of the system, we assume perfect resonance $(\Delta=0)$, and that the decay times $\gamma_{\perp}^{-1}$ and $\gamma_{\|}^{-1}$ are shorter than the decay time of the electric-field $\kappa^{-1}$. We can then apply the adiabatic approximation to the matter Eqs. (29) and (30). That is, we make $\partial_{t} P_{s}=\partial_{t} D_{s}=0$, and insert the result in the field equation (28) to obtain, after a straightforward calculation, the evolution equation of the laser intensity $I \equiv\left|E_{s}\right|^{2}$,

$$
\frac{d I}{d \tau}=\left[-1+\frac{r\left[J_{0}(q)^{2}-J_{2}(q)^{2}\right]}{1+\left[J_{0}(q)+J_{2}(q)\right]^{2} I}\right] I,
$$

where $\tau \equiv 2 \kappa t$ is the dimensionless time and $q=\beta \sqrt{I}$.

\section{A. Analysis of the stationary solutions}

Equation (31) has the trivial stationary solution $I=0$, (no laser emission). If we analyze the stability of this trivial solution, we find that it becomes unstable at $r>1$. The usual laser threshold $r=1$ is then not modified by the presence of the permanent-dipole moments. Above this threshold value, the laser emission takes place and the stationary solution $I_{0}$ verifies

$$
r=\frac{1+\left[J_{0}\left(q_{0}\right)+J_{2}\left(q_{0}\right)\right]^{2} I_{0}}{J_{0}\left(q_{0}\right)^{2}-J_{2}\left(q_{0}\right)^{2}}
$$

with $q_{0}=\beta \sqrt{I_{0}}$. The gain curve given by Eq. (32), i.e., pump versus stationary laser intensity, is shown in Fig. 1. We begin to see multiple stationary laser solutions appear in greater numbers as the pump $r$ becomes larger and larger. The multiplicity in the solutions is induced by the behavior of the Bessel functions, whose presence is due to the difference between the excited and ground permanent-dipole moments. In fact, the different solution branches are separated by the zeros of the term $J_{0}\left(q_{0}\right)^{2}-J_{2}\left(q_{0}\right)^{2}$, as indicated in Eq. (32). In order to obtain a three-valued solution, for example, the value of $q_{0}$ must be greater than approximately four, so that the term $J_{0}\left(q_{0}\right)^{2}-J_{2}\left(q_{0}\right)^{2}$ surpasses the first two zeros. Such a value of $q_{0}$ implies, in general, a large difference between the permanent-dipole moments $\mu_{p}$.

Let us now analyze the stability of the lasing solutions $I_{0}$. Using standard linear-stability methods, we introduce a small perturbation in the solution given by Eq. (32), so that $I=I_{0}$ $+\delta I$. The linearized equation obtained for the perturbation is

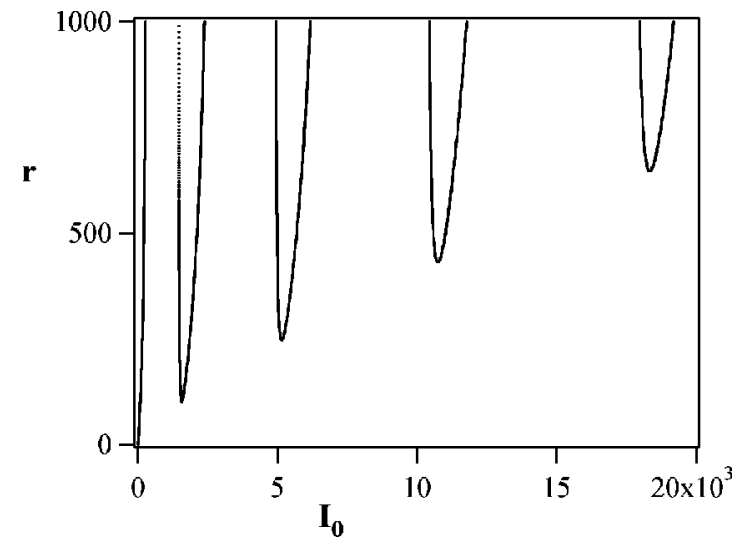

FIG. 1. General shape of the dimensionless pumping $r$ versus stationary dimensionless intensity $I_{0}$, for $\beta=0.1$. Multiple stationary solutions are shown.

$$
\frac{d \delta I}{d \tau}=-\frac{I_{0}}{r}\left[1+\frac{2 r\left[J_{1}\left(q_{0}\right)^{2}-J_{2}\left(q_{0}\right)^{2}\right]}{I_{0}\left[J_{0}\left(q_{0}\right)^{2}-J_{2}\left(q_{0}\right)^{2}\right]}\right] \delta I .
$$

Since the term between the large square brackets in Eq. (33) is the derivative of Eq. (32), that is,

$$
\frac{d r}{d I_{0}}=1+\frac{2 r\left[J_{1}\left(q_{0}\right)^{2}-J_{2}\left(q_{0}\right)^{2}\right]}{I_{0}\left[J_{0}\left(q_{0}\right)^{2}-J_{2}\left(q_{0}\right)^{2}\right]},
$$

we can rewrite Eq. (33) as

$$
\frac{d \delta I}{d \tau}=-\frac{I_{0}}{r}\left(\frac{d r}{d I_{0}}\right) \delta I .
$$

Therefore, the stability of the different lasing solutions $I_{0}$ depends on the sign of the slope $d r / d I_{0}$. When the slope is positive, the perturbation $\delta I$ in Eq. (35) decays to zero and therefore the corresponding solution is stable. On the other hand, when the slope is negative, the perturbation grows exponentially and the solution is then unstable. This is the wellknown criterion in most cases of optical bistability. It is then shown that the presence of permanent dipoles can induce one-photon bistability (or multistability) in lasers.

\section{B. Numerical example}

We now consider: $\omega_{21}=10^{14} \mathrm{~s}^{-1}, \gamma_{\|}^{-1}=10^{-10} \mathrm{~s}$, a shorter value, as usual, for the decoherence time $\gamma_{\perp}^{-1}=10^{-12} \mathrm{~s}$, and $\mu_{p} / \mu_{12}=100$ (e.g., $\left.\mu_{p}=50 \mathrm{D}, \mu_{12}=0.5 \mathrm{D}\right)$. For these values, $\beta \equiv\left(\mu_{p} / \mu_{12}\right)\left(\sqrt{\gamma_{\perp} \gamma_{\|}} / \omega\right) \simeq 0.1$. We recall that $q_{0}$ $=\beta \sqrt{I_{0}}$. The gain curve, i.e., pump versus stationary laser solution, is shown in Fig. 2, where we can see the first three stationary solution branches, only two of them being stable. Therefore, bistability is taking place. For these parameter values, and $q_{0}$ of the order of four (as stated above), the intensity values $I_{0}$ are of the order of $\mathrm{GW} / \mathrm{cm}^{2}$.

The active molecules suitable to show this behavior, must have a large difference between their permanent-dipole moments. These types of molecules are, in general, organic compounds with high polarizabilities, presenting significant 


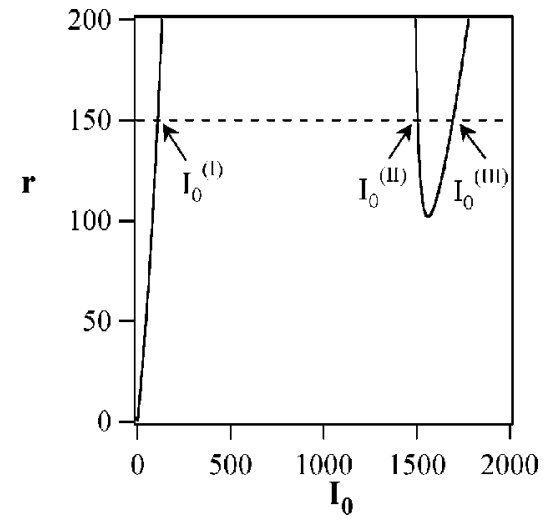

FIG. 2. Dimensionless pumping $r$ versus stationary dimensionless intensity $I_{0}$ for the parameter $\beta=0.1$. For $r=150$, the three stationary solutions $I_{0}^{(I)}, I_{0}^{(I I)}$, and $I_{0}^{(I I I)}$ are shown.

nonlinear optical properties $[12,19]$ and large optical damage thresholds [20].

To observe the bistable behavior of our system, the Eq. (31) is solved numerically with the above-mentioned values and $r=150$. The corresponding three stationary solutions, $I_{0}^{(I)}, I_{0}^{(I I)}, I_{0}^{(I I I)}$, are plotted in a graph (see Fig. 2). The temporal variation of the laser intensity, for different initial conditions, is shown in Fig. 3. The intensity reaches the stable solution $I_{0}^{(I)}$ or $I_{0}^{(I I)}$ depending on the initial condition considered. This result demonstrates the bistable behavior of this system.

Each of the stable solutions (point attractors) is encompassed by a region called a basin of attraction. All the initial conditions $I(\tau=0)$ in the basin, converge on the enclosed point attractor. From the study of basins of attraction in our bistable system, we found that the stationary solution branch with negative slope is the separatrix, delimiting the two basins of attraction.

\section{CONCLUSIONS}

We have studied a single-mode laser system where the two-level active molecules have unequal permanent-dipole moments. As in [3,9], this type of system can be described by means of an effective transition dipole moment depending on the $J_{1}(q)$ Bessel function. The transition is then inhibited at the zeros of $J_{1}$ and enhanced at the maxima of $J_{1}$.

In our case, the argument of the Bessel function is given by $q=\mu_{p} E_{0} /(\hbar \omega)=\left(\mu_{p} / \mu_{12}\right)\left(\sqrt{\gamma_{\perp} \gamma_{\|}} / \omega\right)\left|E_{s}\right|$. It depends on the field amplitude $E_{0}$ (or the normalized amplitude $\left|E_{s}\right|$ ),

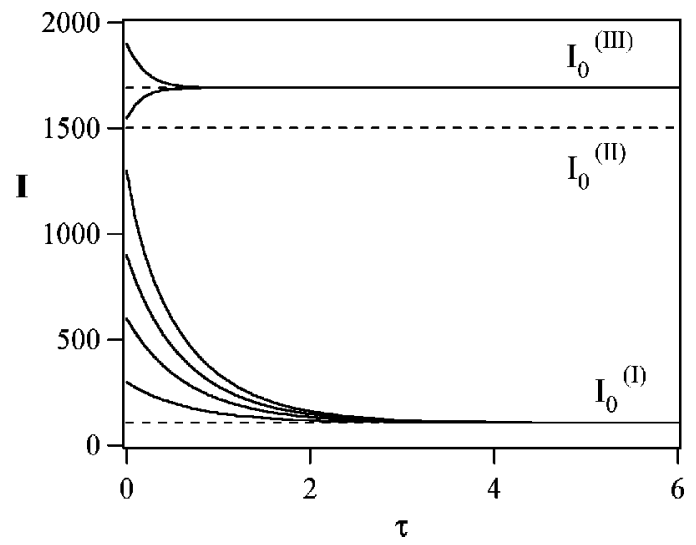

FIG. 3. Dimensionless laser intensity $I$ versus dimensionless time, $\tau \equiv 2 \kappa t$, for different initial conditions. The values of the parameters are $\beta=0.1$ and $r=150$. The two stable solutions $I_{0}^{(I)}$ and $I_{0}^{(I I I)}$, and the unstable one $I_{0}^{(I I)}$, are also shown (dotted lines).

and the difference of the permanent-dipoles $\mu_{p}$. It is found that the Maxwell-Bloch equations are further coupled by nonlinear terms involving Bessel functions of argument $q$. The well-known laser threshold (normalized pumping $r=1$ ) is not modified. However, for higher values of $r$, the new terms can lead to multiple stationary lasing solutions. The multiplicity of the solutions as well as their intensities increase with the pumping.

The linear analysis of the stability shows that the positiveslope regions of the transmitted curves (in our case, pumping versus laser intensity) are stable. It is then shown that the difference between the excited and ground electric permanent-dipole moments can induce multistability in lasers. Organic compounds with large dipole moments and high damage thresholds are suitable for this type of lasers.

For the pumping value $r=150$, three stationary lasing solutions, two of them stable, are found. The ratio between the difference of the dipoles and the transition dipole moment is considered to be 100 . The lasing intensity is then of the order of $\mathrm{GW} / \mathrm{cm}^{2}$. The temporal variation of the intensity, to reach the stable states, is shown numerically for different initial conditions. It is found that the branch of the unstable stationary solution separates the two basins of attraction.

\section{ACKNOWLEDGMENT}

This work was supported by Project No. BFM2000-0796 (Spain).
[1] N. C. Kothari and T. Kobayashi, IEEE J. Quantum Electron. 20, 418 (1984).

[2] T. Hattori and T. Kobayashi, Phys. Rev. A 35, 2733 (1987).

[3] M. A. Kmetic and W. J. Meath, Phys. Rev. A 41, 1556 (1990).

[4] R. Bavli and Y. B. Band, Phys. Rev. A 43, 5039 (1991).

[5] R. Bavli, D. F. Heller, and Y. B. Band, Phys. Rev. A 41, 3960 (1990).

[6] M. A. Antón and I. Gonzalo, J. Opt. Soc. Am. B 8, 1035
(1991).

[7] C. Hoerner, J. P. Lavoine, and A. A. Villaeys, Phys. Rev. A 48, 1564 (1993).

[8] M. C. Bessega, J. L. Paz, A. J. Hernández, and A. E. Cárdenas, Phys. Lett. A 206, 305 (1995).

[9] M. A. Antón and I. Gonzalo, IEEE J. Quantum Electron. 31, 1088 (1995).

[10] V. P. Gavrilenko and E. Oks, Phys. Rev. Lett. 74, 3796 (1995). 
[11] A. E. Kondo and W. J. Meath, Mol. Phys. 92, 805 (1997).

[12] V. A. Kovarsky, B. S. Philipp, and E. V. Kovarsky, Phys. Lett. A 226, 321 (1997).

[13] O. G. Calderón, R. Gutiérrez-Castrejón, and J. M. Guerra, IEEE J. Quantum Electron. 35, 47 (1999).

[14] T. Kobayashi, N. C. Kothari, and H. Uchiki, Phys. Rev. A 29, 2727 (1984).

[15] G. Q. Ge, X. Luo, Y. Wu, and Z. Li, Phys. Rev. A 54, 1604 (1996).

[16] R. G. Harrison, W. Lu, and P. K. Gupta, Phys. Rev. Lett. 63,
1372 (1989).

[17] O. G. Calderón and I. Gonzalo, Opt. Commun. 125, 369 (1996).

[18] O. G. Calderón and S. Melle, in Proceedings of the VIII Spanish Meeting on Statistical Physics, FISES'97, Madrid, Spain, 1997, edited by J. A. Cuesta and A. Sánchez [An. Fís. Monogr. RSEF 4 (1998)].

[19] T. Kobayashi, Mol. Cryst. Liq. Cryst. Sci. Technol., Sect. B: Nonlinear Opt. 314-315, 1 (1998).

[20] K. K. Sharma, Comm. Instr. 3, 145 (1995). 